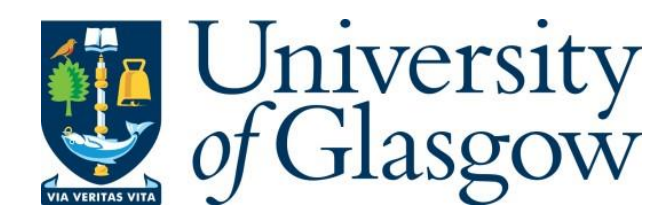

Andry, A. (2021) La lutte oubliée du mouvement syndical pour une réduction du temps de travail en Europe à l'heure du tournant néolibéral = The forgotten labour movement for reduced working hours in Europe during the neoliberal turn. Mouvement Social, 2021(2), pp. 137-152.

There may be differences between this version and the published version. You are advised to consult the publisher's version if you wish to cite from it.

http://eprints.gla.ac.uk/254023/

Deposited on: 6 October 2021

Enlighten - Research publications by members of the University of Glasgow http://eprints.gla.ac.uk 


\title{
La lutte oubliée du mouvement syndical pour une réduction du temps de travail en Europe à l'heure du tournant néolibéral
}

\author{
Aurélie Andry \\ Research Associate en histoire internationale, Université de Glasgow
}

\begin{abstract}
Un projet qui voit dans la dérégulation et la concurrence fiscale et sociale les instruments d'édification d'un continent européen où la promesse est celle de marges de profit toujours croissantes pour les grandes entreprises, de libertés économiques inconditionnelles, et de politiques ne s'attelant qu'à alléger les «fardeaux » réglementaires et réduire les « charges » qui empêchent l'envol des profits. Un projet qui, bien loin d'améliorer leurs conditions de vie et d'emploi, opprime en réalité les peuples et les travailleurs européens ${ }^{1}$.
\end{abstract}

C'est en ces termes que Bernadette Ségol, alors secrétaire générale de la Confédération européenne des syndicats (CES), décrivait il y a quelques années, au cœur de la crise économique et sociale européenne qui suivit le crash financier mondial de 2008, le projet porté par les différents acteurs institutionnels européens ${ }^{2}$. Cette appréciation désabusée de la part d'une des dirigeantes de la plus grande organisation syndicale existant au niveau européen - née il y a près de cinquante ans et censée représenter aujourd'hui environ 45 millions de travailleurs et travailleuses - soulève la question du rôle que le mouvement syndical a pu jouer dans la construction de l'actuelle Europe incarnée par l'Union européenne (UE).

La recherche sur la place des syndicats dans l'histoire de l'intégration européenne, qui s'est développée au cours des deux dernières décennies, ne nous permet pas encore entièrement de répondre à cette question. La plupart des travaux historiques se concentrent en effet principalement sur la reconstruction de la coopération croissante des syndicats au niveau européen ${ }^{3}$ et de leur « européanisation ${ }^{4} »$. Certains ont également retracé ou comparé les stratégies et politiques européennes de différentes centrales syndicales nationales ${ }^{5}$, ou montré les divisions qui ont traversé le mouvement syndical européen au sujet de la « construction » européenne ${ }^{6}$. Peu de recherches permettent cependant de saisir le rôle des syndicats dans la définition, dans une perspective historique, de politiques

\footnotetext{
${ }^{1}$ B. Ségol, « Préface », in C. Degryse et P. Tilly, 1973-2013. 40 ans d'histoire de la Confédération européenne des syndicats, Bruxelles, Institut syndical européen, 2013, p. 5.

${ }^{2}$ Je remercie Claude Roccati, Piers Ludlow ainsi que les membres du groupe de recherche "L'intégration européenne des syndicats » de l'European Labour History Network et de l'équipe du projet ERC « The Making of a Lopsided Union: Economic Integration in the European Economic Community, 1957-1992 » pour leurs conseils lors de la discussion de versions de travail de cet article. Cette recherche a été financée par le Conseil européen de la recherche dans le cadre du programme de recherche et d'innovation Horizon 2020 de l'Union européenne (convention de subvention $\mathrm{n}^{\circ} 716849$ ).

${ }^{3}$ Les exemples les plus connus sont sans doute C. Degryse et P. Tilly, 1973-2013..., op. cit. ; C. Gobin, Consultation et concertation sociales à l'échelle de la Communauté économique européenne. Etude des positions et stratégies de la Confédération européenne des syndicats (1958-1991), Bruxelles, Université Libre de Bruxelles, 1996 ; A. Ciampani et E. Gabaglio, L'Europa sociale e la Confederazione europea dei sindacati, Bologne, Il Mulino, 2010.

${ }^{4}$ Par exemple J. E. Dølvik, ETUC and Europeanisation of Trade Unionism in the 1990s, Oslo, Arena, 1997 ; Q. Jouan, «L'européanisation des syndicats belges et allemands : entre droit, imaginaire et politique (19721985) », thèse de doctorat, Université catholique de Louvain, 2017.

${ }^{5}$ A. Ciampani et P. Tilly (dir.), National Trade Unions and the ETUC: A History of Unity and Diversity, Bruxelles, European Trade Union Institute, 2017 ; I. Del Biondo, L. Mechi et F. Petrini (dir.), Fra mercato comune e globalizzazione: le forze sociali europee e la fine dell'età dell'oro, Milan, Franco Angeli, 2010 ; J.-M. Pernot, «Dedans, dehors, la dimension internationale dans le syndicalisme français », thèse de doctorat en science politique, Université de Nanterre, 2001.

${ }^{6}$ J. Moreno, Trade Unions Without Frontiers: The Communist-Oriented Trade Unions and the ETUC (19731999), Bruxelles, European Trade Union Institute, 2001 ; I. Del Biondo, L'Europa possibile: la CGT e la CGIL di fronte al processo di integrazione europeo, 1957-1973, Rome, Ediesse, 2007.
} 
européennes spécifiques, comme l'union économique et monétaire, la politique sociale, la politique de l'emploi ou la politique commerciale'.

Pourtant, au cours des «longues années 1970 », la gauche ouest-européenne ${ }^{8}$ - en particulier les partis et les syndicats sociaux-démocrates - a déployé des efforts croissants pour s'organiser et se coordonner sur le plan européen, afin de peser davantage sur la définition des politiques européennes. Elle a progressivement formulé un projet visant à changer la nature de l'intégration et de la coopération européennes, et à utiliser les Communautés européennes (CE) comme un outil au service des intérêts des travailleurs en Europe ${ }^{9}$. Ce projet favorisait la redistribution des richesses, la régulation des marchés, la démocratisation économique et celle des institutions européennes, l'harmonisation par le haut des régimes sociaux, la garantie du droit au travail par des politiques de défense de l'emploi : planification économique et sociale, augmentation des dépenses publiques pour satisfaire les besoins collectifs (dans les secteurs de la santé, de l'éducation, du logement, des transports, etc.), contrôle accru des investissements, des grandes entreprises et des sociétés multinationales, et, surtout, réduction du temps de travai $1^{10}$. Souvent désigné à l'époque comme le projet d'une «Europe sociale » ou d'une «Europe des travailleurs », il contrastait fortement avec les formules « néolibérales » qui commencèrent à gagner du terrain dès la fin des années 1970 et avec l'Europe décriée, presque quarante ans plus tard, par Bernadette Ségol et bien d'autres. Qu'est-il donc advenu des propositions pour une Europe sociale?

Pour mieux cerner le rôle (ou le non-rôle) des syndicats européens dans la définition de l'Europe actuelle, cet article explore l'histoire oubliée de la lutte du mouvement syndical européen pour une «Europe des travailleurs » au cours des années 1970 et au début des années 1980, et en particulier ses efforts pour construire à l'échelle européenne un syndicalisme combatif, à même de soutenir ses propositions en matière d'emploi et de réduction du temps de travail. Fondée principalement sur les archives de la CES, des principales centrales syndicales françaises, de plusieurs institutions européennes ainsi que de câbles diplomatiques états-uniens, cette contribution explore tout d'abord l'émergence du projet alternatif d'Europe que les syndicats et leurs alliés de la gauche formulèrent au cours des années 1970, ainsi que le mouvement de construction d'un syndicalisme unitaire et combatif à l'échelle européenne. Dans un deuxième temps, elle retrace la lutte du mouvement syndical européen pour une réduction généralisée du temps de travail en Europe, à travers un double effort de lobbying et de construction (bien que fragile et limitée) d'une mobilisation transnationale des travailleurs et des travailleuses des pays d'Europe occidentale. Enfin, elle s'applique à examiner la mise en échec de cette lutte syndicale européenne inédite et à en évaluer les principales raisons, afin de mieux comprendre l'affirmation d'une autre Europe dans laquelle le droit au travail, la solidarité économique et l'amélioration des conditions de travail et de vie du plus grand nombre devinrent, au mieux, un objectif secondaire.

\section{Vers un mouvement syndical européen combatif pour l'Europe des travailleurs?}

Les « longues années 1968 », qui s'étendirent en fait en Europe jusqu'à la fin des années 1970, constituèrent à bien des égards un âge d'or pour la gauche européenne ; une décennie au cours de laquelle le mouvement syndical, fort d'un renouveau des luttes sociales, apparaissait en mesure d'obtenir des conquêtes historiques. Un peu partout en Europe, les mobilisations ouvrières et étudiantes allaient

\footnotetext{
7 On notera pourtant quelques exceptions, notamment C. Degryse et P. Tilly, 1973-2013..., op. cit.; M. E. Guasconi, « The Origins of the European Social Policy: The Standing Committee on Employment and Trade Unions ", in A. Varsori (dir.), Inside the European Community. Actors and Policies in the European Integration 1957-1972, Baden Baden, Nomos, 2006, p. 301-311.

${ }^{8}$ Dans cet article, j'utilise le terme de "gauche» au sens large pour désigner les formations qui entendent représenter les intérêts des classes ouvrières et populaires : partis de gauche et syndicats principalement, qu'ils se définissent comme « socialistes », « sociaux-démocrates », « travaillistes », « communistes », etc.

${ }^{9}$ Les Communautés européennes rassemblaient la Communauté européenne du charbon et de l'acier (CECA), fondée en 1951, ainsi que la Communauté économique européenne (CEE) et la Communauté européenne de l'énergie atomique (CEAA), fondées en 1957. Une fusion des structures exécutives des trois communautés eut lieu en 1967. Elles formaient l'un des piliers de l'actuelle Union européenne.

${ }^{10}$ Sur le projet d'Europe sociale porté par la gauche ouest-européenne pendant les « longues années 1970 », voir A. Andry, " "Social Europe" in the long 1970s : The Story of a Defeat », thèse de doctorat, European University Institute, Florence, 2017.
} 
de pair avec un important renouveau du féminisme, de l'écologisme, du marxisme, des mouvements antiracistes, antimilitaristes et anti-impérialistes ${ }^{11}$. La contestation sociale et l'impulsion populaire pour davantage de participation à la sphère politique se firent plus intenses que jamais depuis la Seconde Guerre mondiale, et conduisirent dans la plupart des pays à une série de victoires importantes.

Bien que cette recrudescence des mouvements sociaux débordât largement des cadres traditionnels du mouvement ouvrier et posât de nombreux défis à la "vieille » gauche européenne, elle contribua à un renouvellement et un renforcement (momentané) des syndicats et des partis de gauche au cours des années 1970. Les syndicats européens atteignirent ainsi leur apogée, notamment en termes de nombre de membres et de combativité ${ }^{12}$. Sous la pression des militants de base, un mouvement unitaire était par ailleurs engagé au sein du paysage syndical, notamment dans les pays où le mouvement ouvrier avait connu les scissions les plus importantes à l'aube de la guerre froide ${ }^{13}$. En France, la Confédération générale du travail (CGT) et la Confédération française démocratique du travail (CFDT) scellaient en 1966 un accord pour l'unité d'action qui perdura jusqu'à la fin des années $1970^{14}$. En Italie, les trois principales centrales syndicales - la Confédération générale italienne du travail (CGIL), l'Union italienne du travail (UIL) et la Confédération italienne des syndicats de travailleurs (CISL) s'engagèrent en 1972 dans une tentative d'union en une seule Fédération unitaire ${ }^{15}$.

Plusieurs facteurs poussèrent par ailleurs les syndicats européens à envisager davantage de coordination et d'action au-delà du cadre national à partir de la fin des années 1960. Au niveau européen, une nouvelle dynamique des institutions européennes s'engagea après le sommet de La Haye de décembre 1969 qui annonça une « relance » du processus d'intégration européenne au sein des CE. Les nouvelles perspectives qui s'ouvrirent alors - l'élargissement à des pays (le Royaume-Uni et le Danemark en particulier) où la gauche était forte, l'engagement des chefs d'État à développer des politiques sociales et régionales communes, la détermination à influencer l'ordre mondial par le développement d'une politique étrangère commune et de l'Union économique et monétaire - semblaient prometteuses aux yeux de la majeure partie des forces de la gauche européenne, qui ambitionnaient de plus en plus de « changer l'Europe » pour la mettre au service des travailleurs. Par ailleurs, la chute des régimes autoritaires d'Europe méridionale entre 1974 et 1976 créa de nouvelles opportunités pour la gauche en Espagne, au Portugal et en Grèce. Avec la demande d'adhésion de ces pays aux CE, les espoirs de la gauche européenne de peser plus fermement en Europe et sur les CE allaient bientôt $\mathrm{s}^{\prime}$ amplifier ${ }^{16}$. Enfin, le poids grandissant des entreprises multinationales et l'interdépendance de plus en plus visible des économies européennes, surtout après le choc pétrolier de 1973 et la crise économique qui s'ensuivit, poussèrent les forces de gauche à chercher des solutions à l'échelle européenne.

Par conséquent, les syndicats européens s'attelèrent également à accroître leur influence sur les politiques européennes, qui depuis la création de la CEE avait été très limitée ${ }^{17}$. Au tournant des années 1970, il y eut des progrès notoires vers l'unification et la coordination des syndicats au niveau européen. En 1973, la création de la CES marqua un pas important dans cette direction, puisque la Confédération regroupait désormais les syndicats socialistes des neuf pays des CE et des six pays de l'Accord européen

\footnotetext{
${ }^{11}$ Pour un aperçu général des mobilisations en Europe pendant la période, voir par exemple M. Klimke et J. Scharloth (dir.), 1968 in Europe. A History of Protest and Activism, 1956-1977, New York, Palgrave Macmillan, 2008.

${ }^{12}$ Voir par exemple B. Ebbinghaus et J. Visser, Trade Unions in Western Europe Since 1945, Londres-New York, Palgrave Macmillan, 2000 ; M. Pigenet, P. Pasture et J.-L. Robert (dir.), L'apogée des syndicalismes en Europe occidentale, 1960-1985, Paris, Publications de la Sorbonne, 2005.

${ }^{13}$ Sur ces scissions, voir notamment le récent A. Carew, American Labour's Cold War Abroad. From Deep Freeze to Détente, 1945-1970, Edmonton, Athabasca University Press, 2018 ; pour le cas français, A. Lacroix-Riz, « Autour d'Irving Brown : l'AFL, le Free Trade Union Committee, le Département d'État et la scission syndicale française (1944-1947) », Le Mouvement social, n 151, 1990, p. 79-118.

${ }^{14}$ P. Cours-Salies et R. Mouriaux (dir.), L’Unité syndicale en France. 1895-1995, impasses et chemins, Paris, Syllepse, 1996.

${ }^{15} \mathrm{~S}$. Turone, Storia del sindacato in Italia: dal 1943 al crollo del comunismo, Bari, Laterza, 1992.

16 Pour un compte rendu de la chute des régimes dictatoriaux en Espagne, au Portugal et en Grèce et de leur impact sur la gauche européenne, voir D. Sassoon, One Hundred Years of Socialism. The West European Left in the Twentieth Century, Londres, Tauris, 1995, chap. 21.

${ }^{17}$ Leur influence avait été plus importante lors de la création de la CECA, expérience souvent érigée en modèle par certains syndicats comme Force ouvrière. Voir C. Gobin, Consultation et concertation sociales..., op. cit
} 
de libre-échange (AELE). L'année suivante, elle fut rejointe par les syndicats chrétiens de ces pays et même - fait suffisamment important pour être souligné - par l'un des principaux syndicats d'Europe occidentale de tradition communiste : la CGIL italienne ${ }^{18}$. Pour la première fois depuis le début de la guerre froide, des syndicats qui avaient été divisés, au niveau international, entre les trois grandes organisations syndicales mondiales (la Confédération internationale des syndicats libres socialedémocrate, la Confédération mondiale du travail chrétienne et la Fédération syndicale mondiale communiste) se retrouvaient dans une même structure. À la fin de la décennie, l'organisation comptait trente et un syndicats affiliés provenant de dix-huit pays; elle représentait près de 40 millions de travailleurs et de travailleuses. Se plaçant ainsi de très loin comme la principale organisation syndicale au niveau européen, elle pouvait donc espérer peser beaucoup plus lourdement sur les CE et les autres institutions européennes. Dix-huit autres syndicats avaient alors demandé à adhérer à l'organisation, dont la CGT française, les Commissions ouvrières espagnoles (CCOO) et la Confédération générale des travailleurs portugais (CGTP), ainsi que, par exemple, des syndicats turcs, maltais et chypriotes.

Au cours des années 1970, dans un contexte de crise économique et sociale qui voyait le chômage augmenter et la "stagflation"s'installer, la CES déploya des efforts croissants pour définir un programme politique européen commun fondé sur une série de propositions concrètes pour un plan de relance européen coordonné, afin de dépasser la crise et rétablir le plein-emploi. Ses propositions étaient à bien des égards similaires à celles de l' «Europe sociale » promue par les socialistes européens ; elles comprenaient, entre autres, l'extension du secteur public et des investissements dans les services publics, le développement de nouvelles énergies durables, la redistribution des richesses par des mesures fiscales et le contrôle des prix, le contrôle des grandes entreprises, des investissements et des flux financiers, la démocratisation économique, la définition d'un nouvel ordre économique international, etc. Au niveau des CE, la CES demandait la mise en œuvre d'une coordination fiscale et sociale, l'augmentation des fonds sociaux et régionaux, le contrôle des entreprises multinationales, de la spéculation financière et de la fraude fiscale, la création d'une zone européenne de taux de change stables et une politique monétaire commune qui réduise les taux d'intérêt, favorise l'emploi et repose sur un système de solidarité régionale capable de combattre les déséquilibres et les inégalités ${ }^{19}$.

À partir du milieu des années 1970, la CES mena une campagne pour la défense de l'emploi face à ce qu'elle dénonçait comme une inaction de la part des gouvernements européens. Plusieurs syndicats plaidaient alors pour la construction d'un syndicalisme européen plus « combatif », c'est-à-dire qui ne se contente pas de « représenter » les travailleurs auprès des institutions européennes, mais qui constitue une force syndicale réelle capable d'aider à la stimulation et à la coordination des luttes contre le capitalisme européen et mondial. C'était notamment le cas de la CFDT et de FO en France, de la Fédération générale du travail de Belgique (FGTB) ainsi que des syndicats d'Europe méridionale (Grèce, Espagne, Italie, Portugal). Ainsi, le 14 novembre 1975, la CES organisa sa première manifestation européenne pour promouvoir ses revendications. À cette occasion, Edmond Maire, secrétaire général de la CFDT, intervint en ces termes devant les quelques centaines de militants présents :

Il n'est plus de problèmes nationaux qui n'aient une dimension européenne et mondiale. Il n'est plus d'issue totalement satisfaisante à la seule échelle d'un pays. La solution des problèmes revendicatifs les plus immédiats [...] dépend de la vigueur des luttes sociales dans nos différents pays et de la convergence de ces luttes au plan européen, par l'unité d'action de nos confédérations. [...] La CES peut ainsi être un moyen irremplaçable de construction de l'Europe des travailleurs ${ }^{20}$.

\footnotetext{
${ }^{18}$ L'approche unitaire de la nouvelle CES avait été vivement défendue par le TUC britannique, alors que d'autres syndicats s'y étaient opposés. Voir C. Gobin, Consultation et concertation sociales..., op. cit., p. 521-554 ; L. Viannet, «L'activité de la CGT en Europe», in É. Bressol (dir.), La place et les actions de la CGT dans le monde (1945-2000), Montreuil, Institut CGT d'histoire sociale, 2006, p. 111-118.

${ }^{19}$ Institut international d'histoire sociale, Archives de la CES (ACES), 1781, «Communiqué de la CES. Les travailleurs exigent des actions concrètes », 7 février 1978 ; 762, «Rapport d'activités 1976-1978 », mai 1979. Pour un résumé des positions de la CES adoptées lors de chacun de ses congrès, voir $\mathrm{C}$. Gobin, « La Confédération européenne des syndicats. Son programme d'action au fil de ses congrès ", Courrier hebdomadaire du CRISP, $\mathrm{n}^{\circ} 1367-1368,1992$, p. 1-86.

${ }^{20}$ Archives de la CFDT (ACFDT), CH/8/1838, «Intervention E. Maire - meeting CES. Bruxelles le 14 novembre $1975 »$.
} 
La consécration de cette attitude plus combative eut lieu lors du troisième congrès de la CES en mai 1979, à Munich, où quelque deux cents délégués syndicaux adoptèrent à l'unanimité un programme d'action bien plus détaillé et ambitieux qu'auparavant, que certains participants et observateurs décrivirent comme un «New Deal européen ${ }^{21}$ ». Le nouveau Système monétaire européen (SME) créé par les dirigeants des CE ne suffirait pas, selon les syndicats de la CES, à enrayer la crise ${ }^{22}$. Celui-ci devait nécessairement être accompagné d'un mécanisme de redistribution financière entre les pays et s'insérer dans le cadre d'une politique de développement économique "sélectif et planifié », c'est-àdire axé sur le plein-emploi, la satisfaction des besoins collectifs et les préoccupations environnementales, sans quoi il risquerait même d'avoir des conséquences néfastes pour les travailleurs. Le Congrès décida de lancer une campagne de mobilisation coordonnée pour soutenir ces revendications européennes communes, dans tous les pays et au niveau des CE.

Par ailleurs, il élit à la tête de la CES un nouveau président, le Hollandais Willem (Wim) Kok de la Confédération syndicale des Pays-Bas (FNV), qui incarnait un syndicalisme plus « militant» que son prédécesseur allemand, le leader du DGB Heinz Oskar Vetter. Dans son discours de clôture du Congrès, Wim Kok déclara que « le mouvement syndical doit se battre bec et ongles pour que les conséquences de la crise ne retombent pas sur des millions de travailleurs qui ne l'ont pas voulue ${ }^{23} »$. La CES annonçait ainsi haut et fort qu'elle était désormais prête à durcir sa confrontation avec les élites politiques et économiques au niveau communautaire, en organisant des manifestations et en mobilisant l'opinion publique pour soutenir ses revendications, exactement comme tout syndicat national pourrait le faire. Pour appuyer cette annonce, pour la première fois le programme de la CES évoquait la possibilité d'organiser, chaque fois qu'elle le jugerait nécessaire, des « grèves » européennes. Lors des préparatifs du programme d'action, ce sont surtout les syndicats italiens ainsi que la CFDT et Force ouvrière (FO) qui avaient poussé pour un texte et des moyens d'action plus combatifs ${ }^{24}$.

Wim Kok incarnait également le nouveau courant unitaire du syndicalisme européen, et suscitait donc l'espoir de l'émergence d'une CES plus unitaire et donc plus incisive. Avec la perspective encore ouverte d'une possible adhésion de la CGT, voulue un temps par la CFDT, le Trades Union Congress (TUC) et la FGTB et accueillie par une majorité du Comité exécutif de la CES, mais farouchement refusée par les syndicats les plus anti-communistes - en particulier FO, la Confédération allemande des syndicats (DGB) et la CISL -, le mouvement syndical semblait se trouver à un tournant historique ${ }^{25}$. Malgré les péripéties de l'unité d'action, jusqu'en 1980 la CGT resta favorable à la construction d'un syndicalisme européen d'unité et d'action respectueux du pluralisme ${ }^{26}$. Le 4 avril 1979, sa commission exécutive avait ainsi déclaré :

[1]a CGT réitère sa ferme volonté d'adhérer à la CES et souligne la valeur d'une CES forte, indépendante et souveraine, riche de la diversité de ses composantes. Mais, en attendant, elle estime nécessaire de développer l'action unie des travailleurs d'Europe et de leurs organisations syndicales et fera tout pour atteindre cet objectif dans les meilleurs délais ${ }^{27}$.

L'annonce d'un syndicalisme plus fort, plus combatif et plus uni au niveau européen fut perçue à l'époque comme un moment décisif pour le mouvement syndical européen et pour la construction d'une

\footnotetext{
${ }^{21}$ Par exemple «Un "new deal" economico dei sindacati europei. Democrazia in fabbrica e pieno impiego », Avanti !, 16 mai 1979 ; ACES, ETUC-762, « Programme d'action, résolution générale et résolutions spécifiques, 1979-1982 », adopté par le $3^{\mathrm{e}}$ Congrès statutaire, 14-18 mai 1979.

${ }^{22}$ ACFDT, CH/8/1833, « Déclaration de la CES pour les préparatifs du Conseil européen de Brême les 5 et 6 juillet $1978 »$.

${ }^{23}$ ACES, 765, « Closing remarks by Willem Kok ».

${ }^{24}$ ACFDT, CH/8/1834, « Note. Le Congrès de la CES », avril 1979.

${ }^{25}$ La possibilité très concrète de l'entrée de la CGT à la CES jusqu'à son rejet en 1980 est d'ailleurs attestée par les câbles diplomatiques états-uniens. Par exemple, US Mission EC Brussels, «ETUC Executive committee approves CGT talks and reviews East-West conference », 10 février 1975, câbles wikileaks 1975ECBRU01153_b. En ligne : https://wikileaks.org/plusd/cables/1975ECBRU01153_b.html.

${ }^{26}$ Sur ces péripéties et sur la rupture de l'unité d'action, voir C. Roccati, « Si proche, si loin : l'Europe et la CFDT dans la politique revendicative de la CGT », in S. Béroud, É. Bressol, J. Pélisse et M. Pigenet (dir.), La CGT (19751995). Un syndicalisme à l'épreuve des crises, Nancy, Arbre bleu éditions, 2019, p. 299-307.

${ }^{27}$ Archives de la CGT, Archives départementales de Seine-Saint-Denis (ACGT), 242J8, « The problems of Europe. Executive Committee document of April 41979 ».
} 
«Europe des travailleurs ». La presse, surtout celle de gauche, en fit largement état ${ }^{28}$. À la veille de la première élection européenne, ce nouveau syndicalisme européen tombait à point nommé : on espérait que l'émergence d'un mouvement ouvrier transnational pourrait soutenir dans la rue l'alliance des forces progressistes qui pourrait potentiellement sortir de la première élection européenne. Dans son discours de clôture du Congrès, Wim Kok souligna la nouvelle stratégie combative de la CES, qui devait se concentrer en particulier sur une lutte essentielle : la lutte pour une réduction générale du temps de travail au niveau européen ${ }^{29}$.

\section{Réduire le temps de travail : la première lutte transnationale européenne}

À la fin des années 1970 et au début des années 1980, la réduction du temps de travail sans perte de salaire devint le cheval de bataille des syndicats européens pour une stratégie coordonnée de planification du plein-emploi au niveau européen. C'est probablement l'une des revendications sur lesquelles la gauche sociale et politique européenne, qui divergeait sur de nombreuses questions, s'accordait alors le plus unanimement.

Lutte historique du mouvement ouvrier depuis le $\mathrm{XIX}^{\mathrm{e}}$ siècle, la réduction le temps de travail visait d'abord à améliorer les conditions de travail et de vie. Elle était également considérée comme une réponse naturelle aux progrès techniques, qui permettaient d'accroître la productivité du travail et de réduire les besoins de main-d'œuvre. Face à la crise des années 1970 et la montée du chômage qui pesait de manière croissante sur les dépenses sociales, une grande partie de la gauche européenne considéra que la réduction du temps de travail était l'une des clés du rétablissement du plein-emploi et de la stabilité socio-économique en Europe occidentale, puisqu'elle amènerait à la création de nouveaux emplois et donc à une répartition plus équitable du travail disponible ; permettrait ainsi de contenir les dépenses liées aux assurances chômage et d'augmenter les cotisations sociales afin de garantir l'État social ; contribuerait enfin au maintien du « pouvoir d'achat» et donc de la relance économique, ainsi qu'à une plus grande redistribution des fruits de la croissance. Bien que les priorités variassent selon les syndicats et les secteurs, au cours des années 1970, l'objectif de réduction de la durée hebdomadaire du travail et d'allongement des congés payés fut progressivement adopté par la plupart des syndicats ${ }^{30}$.

Lors de son deuxième congrès à Londres, en avril 1976, la CES avait annoncé pour la première fois le lancement d'une campagne européenne pour une réduction du temps de travail au niveau européen. La réduction devait être réalisée par différentes mesures en fonction des situations nationales, combinant réduction du temps de travail hebdomadaire (de préférence à 35 heures), augmentation du nombre de semaines de congés payés (de préférence six), allongement de la période de scolarité (au moins jusqu'à 16 ans), retraite anticipée (de préférence à 60 ans) et limitation des heures supplémentaires.

Compte tenu de l'interdépendance croissante des économies européennes, les syndicats convenaient que, pour être véritablement efficace et éviter des effets indésirables de dumping social, la mesure devait être mise en œuvre de manière coordonnée dans tous les pays d'Europe occidentale. Entre le milieu des années 1970 et le milieu des années 1980, les premiers déployèrent des efforts considérables pour parvenir à un accord au niveau européen en la matière. Au sein des institutions européennes, le projet était principalement soutenu par le commissaire travailliste néerlandais aux Affaires sociales et à l'emploi Henk Vredeling (1977-1981) et par le groupe socialiste du Parlement européen (GSPE). À la fin des années 1970, plusieurs gouvernements semblaient faire preuve d'une certaine ouverture sur la question, au moins sur le plan national : en Belgique, au Danemark, au Luxembourg et en Allemagne de l'Ouest. Le gouvernement italien était quant à lui prudent et affirmait que seul un accord au niveau européen pouvait être efficace en la matière ${ }^{31}$.

\footnotetext{
${ }^{28}$ Par exemple, en Italie, « Concluso a Monaco il congresso della CES. Il neo-presidente annuncia lotte sindacali "con le unghie e coi denti" », Il Messaggero, 19 mai 1979 ; «Storica decisione dei sindacati a Monaco. D'ora in poi lo sciopero può abbracciare l'Europa », Il Giorno, 19 mai 1979.

${ }^{29}$ ACES, ETUC-765, « Closing remarks by Willem Kok ».

${ }^{30}$ ACFDT, CH/8/2056, Institut syndical européen, «La réduction du temps de travail en Europe occidentale », août 1979.

${ }^{31}$ Ibid.
} 
Les dirigeants de la CES crurent initialement en la possibilité de trouver un accord pour restaurer la croissance et l'emploi par le biais de négociations collectives tripartites au niveau européen. Au début des années 1970, plusieurs initiatives avaient vu le jour pour accroître la participation officielle des « partenaires sociaux » au processus décisionnel communautaire, par exemple par la création du Comité permanent de l'emploi (CPE) ; mais sans grands résultats. Entre 1974 et 1978, en réponse à la crise, cinq " conférences tripartites » furent organisées au niveau des CE - comprenant les employeurs, les syndicats, les ministres du Travail et des Affaires économiques et des membres de la Commission - en vue d'établir un «nouveau pacte socio-économique $\mathrm{e}^{32} »$.

Les conférences donnèrent toutefois lieu à une confrontation croissante entre les points de vue des employeurs et des syndicats, tandis que les gouvernements européens se montrèrent réticents à mettre en œuvre les décisions. La CES insistait de plus en plus sur ses propositions de «new deal », auxquelles les revendications des organisations patronales, en particulier l'Union des confédérations des industries et des employeurs d'Europe (UNICE), étaient diamétralement opposées. Celles-ci considéraient que les salaires trop élevés étaient la principale raison de l'inflation et exigeaient la maîtrise des salaires et une plus grande flexibilité du marché du travail comme condition pour rétablir la croissance, donc l'emploi ; elles demandaient une libéralisation du marché et une moindre intervention des pouvoirs publics dans la gestion économique, mais invoquaient des politiques d'aide publique à l'investissement des entreprises et de réduction des charges sociales et fiscales; enfin, elles se montraient particulièrement sceptiques à l'égard de la proposition de réduction du temps de travail qui, selon elles, mettrait en péril la croissance et donc détruirait des emplois. La conférence de novembre 1978, censée examiner la question, finit dans l'impasse, car les employeurs s'opposaient fermement aux demandes des syndicats pour un accord-cadre européen sur la réduction du temps de travail et les gouvernements semblaient peu disposés à prendre des décisions fermes sur la question. Peu après, la CES annonça qu'elle ne participerait plus aux conférences tripartites dans ces conditions.

Le mouvement syndical européen, soutenu par ses alliés de la gauche européenne, continua néanmoins de relancer ses propositions de dialogue, bien qu'il se heurtât à la nette opposition de l'UNICE qui refusa de participer à une série de conférences tripartites sur la réduction du temps de travail : celle de juin 1978 au niveau de l'AELE et de la CEE, celle de mars 1980 organisée au niveau du Conseil de l'Europe, celle proposée en mai 1980 par Vredeling. Plus tard, le nouveau président de l'UNICE, Guido Carli, ancien directeur général de la Banque centrale italienne et promoteur du SME, refusa les propositions de Vredeling d'engager des négociations sur des accords-cadres entre l'UNICE et la CES, et n'accepta de s'engager dans un échange d'informations en vue d'une limitation du temps de travail annuel qu'en échange de compromis en termes de flexibilité de la main-d'œuvre, afin que la productivité ne soit pas menacée ${ }^{33}$.

Malgré ces difficultés, la lutte du mouvement syndical se poursuivit. À la fin des années 1970 et au début des années 1980, les syndicats européens et leurs alliés firent pression pour que la Commission européenne et le Conseil prennent un engagement politique sur la nécessité d'une stratégie coordonnée de planification économique et sociale pour surmonter la crise, et en particulier pour une réduction du temps de travail afin de protéger l'emploi. Le 9 février 1978, les dirigeants de la CES rencontraient le nouveau président de la Commission, le travailliste britannique Roy Jenkins, et lui faisaient part de leur scepticisme quant à son projet d'Union économique et monétaire tant que celui-ci ne s'inscrirait pas dans une perspective de création d'emplois. Georges Debunne, secrétaire général de la FGTB, expliqua que la priorité devait être à la réduction du temps de travail, pas à l'union monétaire qui ne résoudrait pas les problèmes des travailleurs ${ }^{34}$.

Le 5 avril 1978, la CES organisa, pour la première fois, une « journée d'action européenne pour le plein-emploi » dans tous les pays d'Europe occidentale. Conférences de presse, manifestations dans de nombreuses villes européennes, rencontres avec les gouvernements, les CE, l'AELE, le Conseil de

\footnotetext{
32 A. Becherucci, «Prove di dialogo sociale: la CEE e le Conferenze tripartite degli anni settanta », in I. Del Biondo, L. Mechi et F. Petrini (dir.), Fra mercato comune e globalizzazione ..., op. cit., p. 179-202.

${ }^{33}$ C. Gobin, Consultation et concertation sociales..., op. cit., p. 461-519 ; L. Warlouzet, « L'Europe occidentale face au choc de la globalisation. La solution de la CEE (1973-1985) », mémoire d'habilitation à diriger des recherches, Université de Rouen, 2015, p. 153.

${ }^{34}$ US Embassy Brussels, « European Labor and European monetary union », 15 février 1978, câble wikileaks. En ligne : https://wikileaks.org/plusd/cables/1978BRUSSE02960_d.html.
} 
l'Europe, le Conseil européen et les organisations patronales eurent un large écho dans la presse européenne ${ }^{35}$. En Italie, en Espagne, en Grèce et en Belgique, pour la première fois, des grèves « européennes » étaient organisées par les syndicats; des grèves spontanées se déclenchèrent en Finlande $^{36}$. Selon la CCOO et l'Union générale des travailleurs, en Espagne, 3720600 personnes participèrent aux actions (soit près de $50 \%$ des travailleurs salariés espagnols), dont 800000 à Madrid, 980000 en Catalogne et 514000 en Andalousie ${ }^{37}$. Bien que la participation n'ait pas eu la même intensité dans tous les pays, la CES s'estimait très satisfaite de cette première démonstration de force. Quelques jours plus tard, au Conseil européen de Copenhague, les chefs d'État donnèrent des signes relativement encourageants lorsqu'ils s'engagèrent, pour progresser vers l'Union économique et monétaire, et redresser la situation économique et sociale, à élaborer une stratégie commune couvrant les affaires économiques et monétaires, l'emploi, l'énergie, le commerce, les affaires industrielles et les relations avec le monde en développement ${ }^{38}$.

Au sein de la Commission européenne, Vredeling poussait dans la même direction. Entre 1977 et le début de 1981, la Direction générale Affaires sociales (DG V), qu'il dirigeait, réalisa une série d'études qui mettaient en évidence les effets bénéfiques de la réduction du temps de travail en termes de création d'emplois, de bien-être et de productivité. Il organisa plusieurs réunions informelles avec les dirigeants des syndicats affiliés à la CES, en juin 1978 et février 1979, pour discuter de la meilleure stratégie possible pour parvenir à un accord européen sur le temps de travail. Les syndicats redoublèrent d'efforts pour faire pression sur les autorités gouvernementales nationales de leurs pays respectifs, d'autant plus qu'au cours du premier semestre 1979, sous la présidence française, des négociations furent entamées au Conseil à ce sujet. Les syndicats français firent pression sur leur gouvernement, tout comme les dirigeants de la CES $^{39}$. En mars 1979, la CFDT organisa à Paris une « table ronde eurosyndicale » sur la réduction du temps de travail, en présence de militants et de journalistes, à laquelle participèrent Edmond Maire et Albert Mercier (CFDT), le président de la CES, Wim Kok (FNV), Aldo Bonaccini (CGIL), Eugen Loderer (DGB), Georges Grinberg (FGTB) et François Staedelin (CES); elle fut suivie d'une période d'action pour une réduction du temps de travail lancée par la CFDT et suivie par la CGT, du 2 au 14 avril $1979^{40}$.

Les efforts déployés au sein des institutions européennes et auprès des gouvernements donnèrent toutefois des résultats très décevants pour les syndicats. Le 15 mai 1979, la réunion très attendue du Conseil des ministres des Affaires sociales des $\mathrm{CE}$, qui devait enfin discuter de la réduction du temps de travail, suscita une vive déception car elle se contenta de charger une fois de plus la Commission européenne de réaliser de nouvelles études sur la question. Entre-temps, la Commission, malgré ses travaux, ne prenait pas fermement position en faveur de la réduction du temps de travail. Le premier document qu'elle produisit, discuté dans le cadre du CPE, n'aboutit à aucun accord entre ses participants, les représentants des employeurs ayant refusé ici aussi de négocier sur la question ${ }^{41}$. Le 22 mai 1979, lors d'une réunion du CPE, la CES menaça de suspendre sa participation aux discussions tant qu'un engagement ferme n'aurait pas été pris pour parvenir à un accord. Par conséquent, le ministre français du Travail, alors président en exercice du Conseil, Robert Boulin, affirma que le Conseil était déterminé à obtenir des résultats concrets et réels au plus tard le $1^{\mathrm{er}}$ décembre suivant. La délégation des employeurs

\footnotetext{
${ }^{35}$ ACES, 1771, « Première brève évaluation de la journée d'action du 5 avril 1978 », 20 avril 1978.

${ }^{36}$ ACES, 1773.

${ }^{37}$ ACES, 1777.

38 Conclusions du Conseil de Copenhague, 7-8 avril 1978. En ligne : https://www.consilium.europa.eu/media/20770/copenhagen_avril_1978_fr_pdf.

${ }^{39}$ Archives de Force ouvrière (AFO), boîte CES-1/4, « La CES à 1’Élysée. En attendant la semaine européenne de 35 heures », FO Hebdo, 13 mars 1979 ; « Durée du travail : grèves et manifestations aujourd'hui », Le Matin, 29 novembre 1979 ; ACFDT, CH/8/1834, Hinterscheid à Giscard d'Estaing, 9 mai 1979 ; Hinterscheid à Boulin, 30 mai 1979 ; « Circulaire. Entrevue de la CES avec le Président en exercice des CE Valéry Giscard d'Estaing », 13 juin 1979.

${ }^{40}$ ACFDT, CH/8/1838. Dans un contexte de détérioration de l'accord unitaire CGT-CFDT, la CGT, qui avait demandé de participer à cette table ronde européenne, en fut cependant exclue. FO refusa de participer à cette quinzaine de mobilisation.

${ }^{41}$ Archives historiques de la Commission européenne (AHCE), 375/1999-1342, « La répartition du travail objectifs et effets »; " Secrétariat Général, note à l'attention de MM. les membres de la Commission. 13e réunion du Comité Permanent de l'emploi tenue à Bruxelles le 21 mars 1978 sur la division du travail ».
} 
déclara cependant qu'elle refusait d'entamer des négociations au niveau européen ${ }^{42}$. La prochaine réunion du Conseil des CE pour les Affaires sociales et le prochain sommet du Conseil européen étaient tous deux prévus pour le mois de novembre.

Dans ce contexte, les syndicats européens continuèrent de faire campagne plus résolument que jamais pour promouvoir leurs propositions aux niveaux local, national, européen et international. Fin novembre 1979, en vue du Conseil européen de Dublin et conformément à sa nouvelle position combative affirmée au congrès de Munich, la CES organisa une «semaine européenne d'actions » incluant manifestations, conférences de presse, initiatives dans les principales villes européennes avec échange d'intervenants entre les organisations syndicales de différents pays, diffusion de brochures et d'affiches portant des slogans communs, réunions avec les gouvernements et les organisations d'employeurs et, parfois, actions de grève. Le moment semblait particulièrement stratégique puisque les syndicats espéraient forcer les dirigeants européens à prendre enfin, comme promis, une décision ferme sur le temps de travail, à un moment où même les relations avec la Commission s'étaient dégradées : dans le contexte économique de plus en plus tendu du second choc pétrolier, la CES accusait en effet la Commission d'adopter les vues des employeurs et des gouvernements, et de favoriser la lutte contre l'inflation au détriment de l'emploi et donc de millions de travailleurs ${ }^{43}$. La semaine d'action européenne du 24 au 30 novembre 1979 fut largement suivie en Grèce, Italie, France, Suisse, Autriche, Allemagne, Irlande, Belgique, ainsi que dans les pays scandinaves, au Luxembourg, à Malte, aux Pays-Bas et en Espagne, où les réunions avec les autorités publiques furent accompagnées d'actions et de manifestations publiques, et parfois de grèves ${ }^{44}$.

En France, où la lutte pour la réduction du temps de travail était une priorité syndicale, tous les syndicats se mobilisèrent lors de la semaine d'action européenne, y compris la CGT qui appela ses affiliés, avec la CFDT et la FEN, «à prendre les initiatives les plus larges possibles [...] sous forme d'arrêts de travail et de manifestations en vue d'étendre l'action au plus grand nombre de travailleurs ${ }^{45}$ ". L'appel unitaire fut largement suivi : les fédérations PTT de la CGT et de la CFDT appelèrent à participer en masse aux initiatives, notamment par une grève des services financiers le 22 novembre et une grève des bureaux de poste, des centres de tri et des installations de télécommunications le 29 novembre; les mêmes instructions furent données pour une participation massive, entre autres, des cheminots et des employés de banque ; en Aquitaine, les syndicats régionaux annoncèrent un arrêt d'au moins deux heures et des manifestations. Le 29 novembre, des manifestations eurent lieu à Paris et dans plusieurs autres villes comme Marseille, Bordeaux, Lille et Clermont-Ferrand ${ }^{46}$. FO, quant à elle, refusa de participer aux initiatives unitaires mais appela ses fédérations et unions départementales à suivre les instructions de la CES pour organiser des actions auprès des employeurs, du gouvernement et des pouvoirs publics à tous les niveaux, et à soutenir ces actions par toutes les manifestations jugées appropriées ainsi que par un arrêt de travail d'au moins une heure le 30 novembre en fin de journée ${ }^{47}$. L'appel fut plutôt suivi : La Fédération FO Chemins de fer appela à un arrêt de travail d'une heure le 28 novembre ; la Fédération FO Finances décida d'une grève générale de 24 heures ; FO Électricité et Gaz appela à des arrêts de travail d'une heure en fin de journée les 27, 28, 29 et 30 novembre; etc. ${ }^{48}$. Une délégation de FO, conduite par son secrétaire André Bergeron, fut reçue à Matignon le 26 novembre par le Premier ministre Raymond Barre et le nouveau ministre du Travail Jean Mattéoli, pour parler avant tout de la réduction du temps de travail ${ }^{49}$.

La semaine européenne de mobilisation de novembre 1979, comme la journée d'action d'avril 1978, constituèrent certainement une grande nouveauté dans l'histoire de la mobilisation des travailleurs en

\footnotetext{
${ }^{42}$ ACFDT, CH/8/1834, « Circulaire. Réunion du Comité Permanent de l'emploi du 22 mai 1979 », 29 mai 1979.

${ }^{43}$ Fin 1979, un dirigeant de la CFDT décrivait Vredeling comme un personnage " pâteux », « souvent plus proche des positions patronales par prudence tactique que de celles de la CES ». ACFDT, CH/8/1838, « Compte rendu rencontre CES-CEE-UNICE 7 et 8 novembre $1979 »$, par Michel Rolant, 12 novembre 1979.

${ }^{44}$ ACFDT, CH/8/1834, « Premières informations sur la semaine d'action », 26 novembre 1979.

45 « La FEN dans l'action du 26 au 30 novembre », Le Matin, 21 novembre 1979.

46 « Nouvelle action commune la semaine prochaine », L'Humanité, 21 novembre 1979.

${ }^{47}$ AFO, boîte CES 1/4, « Circulaire. Semaine d'action européenne 24-30 novembre 1979 », 6 novembre 1979 ; «Communiqué. FO demande à ses militants et adhérents de cesser le travail au moins une heure le 30 novembre $1979 », 21$ novembre 1979.

48 « La semaine d'action des travailleurs européens », FO Hebdo, 28 novembre 1979.

49 « Durée du travail : grèves et manifestations aujourd'hui », Le Matin, 29 novembre 1979.
} 
Europe et témoignèrent d'une phase d'activisme particulièrement incisive pour les syndicats européens.

\section{Réduction ou réorganisation du temps de travail ? La défaite progressive}

Tout cela ne suffit cependant pas à convaincre les gouvernements européens. Au Conseil, en effet, les gouvernements des États membres ne soutinrent jamais résolument le projet de réduction du temps de travail poussé par les syndicats. Le gouvernement ouest-allemand de Schmidt s'était d'abord montré ouvert mais resta ambigu sur la question. Le gouvernement français de Valéry Giscard d'Estaing semblait sensible aux discussions sur l'Europe sociale et le temps de travail, mais sa conception du problème différait en tous points du projet présenté par la gauche européenne : il refusait toute réduction du temps de travail sans perte de salaire et préférait des solutions comme la limitation des heures supplémentaires ou l'encouragement du travail à temps partiel (ce qui revenait à augmenter la flexibilité du travail). Il préférait donc le terme « réorganisation » à celui de "réduction » du temps de travail, moins contraignant et plus compatible avec des objectifs de «flexibilisation» du travail. Le gouvernement italien, sous la pression des syndicats italiens, était disposé à mettre la question à l'ordre $\mathrm{du}$ jour mais ne montra jamais un soutien résolu au projet. Alors que le gouvernement travailliste britannique avait été prudent sur la question, le gouvernement conservateur de Thatcher, au pouvoir en mai 1979, s'opposa farouchement au projet ${ }^{50}$.

Le résultat fut une résolution très modeste (et non contraignante) sur le temps de travail adoptée par le Conseil des Affaires sociales des CE le 22 novembre 1979. Les États membres, réunis à Dublin la semaine suivante, ne prirent aucune décision sur la réduction du temps de travail et déclarèrent au contraire que la priorité devait être donnée à la lutte contre l'inflation et non, comme le demandaient les syndicats, contre le chômage.

Malgré tous les efforts de lobbying et la semaine d'actions de protestation organisée à cette occasion - et en dépit des engagements pris précédemment par les gouvernements -, les syndicats européens et autres promoteurs du projet perdirent la bataille. Ils furent très déçus par les décisions du Conseil et accusèrent les gouvernements de soutenir les demandes et les intérêts du patronat au détriment des travailleurs européens, des 9 millions de chômeurs, de tout espoir de paix sociale et de démocratie durables en Europe. La CES renouvela son appel à poursuivre et intensifier les actions et la mobilisation en faveur de ses propositions ${ }^{51}$.

Au cours des années suivantes, la CES poursuivit sa tentative de construire un syndicalisme d'action au niveau européen, bien que de manière moins ambitieuse. En juin 1980, lors du Conseil européen, la CES rassembla à Venise une manifestation de 5000 militants ; une autre manifestation eut lieu en mars 1981 lors du Conseil de Maastricht; en juin 1981, la CES organisa, à l'occasion du Conseil de Luxembourg, une manifestation à laquelle participèrent 12000 personnes et où elle présenta son manifeste intitulé «Pour l'emploi et la reprise économique »; en février 1983, elle organisa un festival pour le dixième anniversaire de sa création à Bruxelles, où 5 à 6000 militants se rassemblèrent pour une manifestation; en 1983, elle lança une vaste campagne européenne pour l'emploi, renforcée en juin par une importante manifestation européenne organisée avec le soutien de la DGB à Stuttgart, à laquelle participèrent 80000 personnes. Le tournant des années 1980 fut sans aucun doute la période la plus combative de l'histoire du syndicalisme au niveau européen, du moins jusqu'aux années 2000, mais récolta peu de fruits ${ }^{52}$.

La bataille dura cependant plusieurs années encore. Après 1981, sous la présidence de François Mitterrand, le nouveau gouvernement socialiste français tenta de remettre la question à l'ordre du jour

\footnotetext{
${ }^{50}$ Pour une description détaillée des positions des États membres sur la question, voir L. Warlouzet, « L’Europe occidentale face au choc de la globalisation... », op. cit., p. 93-99.

${ }^{51}$ ACFDT, CH/8/1834, « Résolution sur les conclusions en matière d'aménagement du temps de travail du Conseil des ministres des Affaires sociales », 30 novembre 1979; «Déclaration de la CES sur le Conseil européen de Dublin », 5 décembre 1979.

52 Voir C. Degryse et P. Tilly, 1973-2013 ..., op. cit., p. 239-241 ; G. Debunne, Les syndicats et l'Europe. Passé et devenir, Bruxelles, Éditions Labor, 1987, p. 141-158.
} 
des $\mathrm{CE}^{53}$. La Commission - où le successeur de Vredeling à la tête de la $\mathrm{DG} \mathrm{V}$, le travailliste britannique Ivor Richard, était peu disposé à agir pour la réduction du temps de travail - réalisa de nouvelles études, qui furent examinées par le Comité de politique économique, mais sans résultats ${ }^{54}$. Au Conseil, l'hostilité du gouvernement Thatcher était renforcée par la réticence du gouvernement Schmidt et, après octobre 1982, par celle du nouveau gouvernement de Helmut Kohl, aux prises avec la lutte des travailleurs allemands concernant la semaine de 35 heures et désireux de tenir les CE à l'écart de cette question. Le gouvernement britannique mit son veto à la proposition de recommandation de la Commission lors de la réunion du Conseil des Affaires sociales du 7 juin 1984. Les neuf autres États membres décidèrent alors d'adopter une recommandation sur la réduction et l'aménagement du temps de travail, mais une fois de plus, elle se montrait très prudente, non contraignante et envisageait la « réorganisation» du temps de travail comme un moyen d'accroître la flexibilité du travail et la compétitivité - à l'opposé de la réduction générale du temps de travail de $10 \%$ sans perte de salaire soutenue par la gauche européenne ${ }^{55}$.

Les raisons de cette défaite sont évidemment multiples. Certaines étaient " exogènes » aux syndicats européens : le refus obstiné du patronat européen; l'essoufflement de l'élan social-démocrate des années 1970 au profit de la droite libérale et conservatrice; la popularité croissante des politiques d'austérité et néolibérales parmi les élites politiques et économiques, qui finirent par gagner une partie de la gauche européenne ; la détérioration, consécutive à ce virage idéologique, des liens de la CES avec la Commission européenne et des connivences des centrales syndicales avec certaines formations sociales-démocrates. Il est vrai qu'au tournant des années 1980, quand fut lancée l'offensive de la CES, la fenêtre d'opportunité qui s'était ouverte pendant les années 1970 était déjà en train de se refermer, l'élan unitaire et combatif du mouvement syndical s'essoufflait, et le processus de désyndicalisation était enclenché. Un nouvel ordre émergeait, qui consacrait l'accroissement de la concurrence, la libéralisation des marchés, les privatisations, les délocalisations et la déréglementation, et conduisait à une augmentation des inégalités ${ }^{56}$.

Mais certaines raisons étaient aussi " endogènes » et concernaient les caractéristiques et l'attitude des syndicats européens : quelques désaccords persistants entre syndicats sur des questions socioéconomiques et sur l'intégration européenne; des divergences stratégiques entre une approche « contractuelle » (FO, TUC) et une approche "réglementaire » privilégiant une régulation au niveau communautaire (DGB, CFDT), ainsi qu'entre un syndicalisme de mobilisation et un syndicalisme d'institutionnalisation et de concessions ${ }^{57}$; l'échec de la construction d'un syndicalisme européen véritablement unitaire en raison du refus constant de certains syndicats (DGB et FO en tête) d'inclure les syndicats communistes dans la $\mathrm{CES}^{58}$; l'échec de la construction d'un mouvement de masse transnational véritablement combatif au niveau européen.

Les dirigeants syndicaux d'Europe occidentale ont-ils vraiment lutté «bec et ongles» pour la réduction du temps de travail, alors qu'ils demandaient à leurs affiliés de se mobiliser massivement en 1978 et 1979 ? Il est possible d'en douter. À en croire les câbles diplomatiques américains, au congrès de Munich, alors que Georges Debunne (FGTB) insistait pour l'adoption des « 35 heures » comme slogan commun de tous les syndicats en Europe, plusieurs d'entre eux, en particulier nordiques, s'opposaient à cette proposition, arguant qu'il fallait rester flexibles sur les revendications. Par ailleurs,

\footnotetext{
${ }^{53}$ "Mémorandum du gouvernement français sur la relance européenne », 13 octobre 1981. En ligne : http://www.cvce.eu/content/publication/2002/10/16/a8377c45-380f-4679-8d17$62 \mathrm{~d} 42 \mathrm{f} 4734 \mathrm{~d} 5 /$ publishable fr.pdf.

${ }^{54}$ AHCE, 375/1999-1929, «Document interne de la Commission sur les travaux du Comité de politique économique » (non daté).

${ }^{55}$ Voir L. Warlouzet, «L'Europe occidentale face au choc de la globalisation... », op. cit., p. 95-99.

${ }^{56}$ Voir, par exemple, T. Piketty, Le capital au XXI siècle, Paris, Éditions du Seuil, 2013.

${ }^{57}$ G. Groux, R. Mouriaux et J.-M. Pernot, «L'européanisation du mouvement syndical : la Confédération européenne des syndicats », Le Mouvement social, $\mathrm{n}^{\circ}$ 162, 1993, p. 57.

${ }^{58}$ Les archives montrent, outre l'opposition féroce de FO, le sabotage probablement volontaire de la part de Vetter (DGB), alors président de la CES, contre l'entrée de la CGT à la CES et sa participation à la journée d'action européenne d'avril 1978, alors que la CGT engageait des efforts considérables pour faire avancer sa candidature à la CES. AFO, boîtes CES $1 / 3$ et $1 / 4$; ACFDT, CH/8/1833, « Réunion du Comité exécutif de la CES 9 et 10 février 1978. Informations et suites à donner », 15 février 1978; ACFDT, CH/8/1833, « Réunion du Comité exécutif de la CES, 13-14 avril $1978 », 20$ avril 1978.
} 
plusieurs leaders syndicaux nordiques auraient confié au Labour Attaché des États-Unis auprès des CE, lors de conversations privées en marge du congrès, qu'ils avaient des doutes quant à l'opportunité et au réalisme de la réduction du temps de travail ${ }^{59}$. En effet, la synthèse " travailler tous et vivre mieux » cachait en fait un désaccord de fond: certains syndicats mettaient prioritairement en avant cette revendication comme créatrice d'emplois, alors que d'autres, principalement des pays nordiques, étaient sceptiques et adhéraient à cette revendication plutôt comme source d'amélioration des conditions de vie et de travail. D'autre part, malgré les annonces du congrès de Munich, les syndicats au sein de la CES ne furent jamais à même d'appeler à de véritables grèves générales européennes coordonnées, et ce jusqu'à nos jours. La semaine de mobilisation de novembre 1979 marqua certainement un effort important pour construire un mouvement syndical européen, mais là aussi, la proposition d'organiser une grève coordonnée dans toute l'Europe fut rejetée par une majorité de syndicats au sein du Comité exécutif de la $\mathrm{CES}^{60}$.

La victoire progressive du concept de «réorganisation» du temps de travail sur l'idée de « réduction» préfigurait en quelque sorte la victoire graduelle du projet néolibéral sur le projet d'« Europe sociale ». Au cours des années 1980, avec la création du marché unique européen et malgré le lancement du nouveau « dialogue social européen » sous l'impulsion de Jacques Delors, les initiatives communautaires dans les domaines de la politique sociale et de l'emploi s'orientèrent de plus en plus vers des objectifs de compétitivité et de flexibilité. Plusieurs autres propositions aboutirent à la même impasse et à des résultats similaires : ainsi, les efforts pour augmenter le budget des CE pour les politiques de redistribution, pour accompagner le SME d'un mécanisme de solidarité financière entre les pays, pour faire passer une directive sur la consultation des travailleurs dans les grandes entreprises multinationales, etc. Malgré quelques avancées dans le domaine social - par exemple, en ce qui concerne l'égalité de traitement entre hommes et femmes ou la santé et la sécurité au travail -, il était clair, à partir du milieu des années 1980, que les tentatives de mise en œuvre d'une « Europe sociale » axée sur la redistribution et la régulation du marché avaient été écartées ${ }^{61}$.

Bien qu'elle ait joué un rôle crucial dans l'élaboration de positions communes et la création d'une culture syndicale commune - contribuant ainsi à l'« européanisation » du syndicalisme européen -, la CES reste jusqu'à aujourd'hui un organe de représentation au sein des institutions plutôt que de lutte. Contrairement aux attentes de la fin des années 1970, faute de volonté politique de la plupart des confédérations nationales qui la composent, dépourvue de moyens, elle demeure toujours un colosse aux pieds d'argile, qui ne parvint jamais à construire ce syndicalisme européen unitaire et combatif que les travailleurs et travailleuses appelaient alors de leurs vœux afin de construire un rapport de force suffisant pour imposer « l'Europe des travailleurs ».

\footnotetext{
${ }^{59}$ US Embassy Brussels, «ETUC Congress May 14-18: a more militant posture on reducing working time », 22 mai 1979, câble wikileaks 1979BRUSSE09335_e. En ligne : https://wikileaks.org/plusd/cables/1979BRUSSE09335_e.html

${ }^{60}$ AFO, boîte CES-1/4, « Circulaire. Semaine d'action européenne 24-30 novembre 1979 », 6 novembre 1979. FO avait préconisé pour l'organisation un arrêt de travail d'une heure dans toute l'Europe, mais la proposition n'obtint pas un soutien majoritaire.

${ }^{61}$ Notons cependant que cette revendication ne fut pas complètement abandonnée par la CES (qui l'a d'ailleurs réaffirmée lors de son dernier congrès à Vienne), mais fut noyée au sein d'autres revendications sans claire priorité et cantonnée au niveau des négociations collectives. Voir U. Rehfeldt, «Le Congrès de Vienne de la CES : une confédération plus unie mais avec moins d'adhérents ", Chronique internationale de l'IRES, ${ }^{\circ} 167,2019$, p. 37-49.
} 